\title{
Habituation, latent inhibition, and extinction
}

\author{
Wesley P. Jordan • Travis P. Todd • David J. Bucci • \\ Robert N. Leaton
}

Published online: 13 February 2015

(C) Psychonomic Society, Inc. 2015

\begin{abstract}
In two conditioned suppression experiments with a latent inhibition (LI) design, we measured the habituation of rats in preexposure, their LI during conditioning, and then extinction over days. In the first experiment, lick suppression, the preexposed group (PE) showed a significant initial unconditioned response (UR) to the target stimulus and significant long-term habituation (LTH) of that response over days. The significant difference between the PE and nonpreexposed (NPE) groups on the first conditioning trial was due solely to the difference in their URs to the conditioned stimulus (CS) - a habituated response (PE) and an unhabituated response (NPE). In the second experiment, bar-press suppression, little UR to the target stimulus was apparent during preexposure, and no detectable LTH. Thus, there was no difference between the PE and NPE groups on the first conditioning trial. Whether the UR to the CS confounds the interpretation of LI (Exp. 1) or not (Exp. 2) can only be known if the UR is measured. In both experiments, LI was observed in acquisition. Also in both experiments, rats that were preexposed and then conditioned to asymptote were significantly more resistant to extinction than were the rats not preexposed. This result contrasts with the consistently reported finding that preexposure either produces less resistance to extinction or has no effect on extinction. The effect of stimulus preexposure survived conditioning to asymptote and was reflected directly in extinction. These two experiments provide a cautionary procedural note for LI experiments and have shown an unexpected extinction effect that may provide new insights into the interpretation of LI.
\end{abstract}

W. P. Jordan $(\bowtie)$

Psychology Department, St. Mary's College of Maryland,

18952 E. Fisher Road, St. Mary's City, MD 20686, USA

e-mail: wpjordan@smcm.edu

T. P. Todd $\cdot$ D. J. Bucci $\cdot$ R. N. Leaton

Dartmouth College, Hanover, New Hampshire, USA
Keywords Latent inhibition $\cdot$ Habituation $\cdot$ Extinction $\cdot$ Fear conditioning

Repeated presentations of a novel stimulus lead to a reduction of the unconditioned response (UR) to that stimulus, a process known as habituation. Habituation, both short-term (STH) and long-term (LTH), has been demonstrated in a variety of species and response systems (see Rankin, 2009; Tighe \& Leaton, 1976, for reviews.) This same stimulus presentation procedure also produces latent inhibition (LI). In the LI paradigm, the habituation series is called preexposure, and subsequent conditioning to the preexposed stimulus is degradedthat is, conditioning is latently inhibited. LI has been demonstrated in a variety of species and response systems (see Lubow \& Weiner, 2010b, for reviews).

Several investigators have acknowledged these parallels and measured habituation during the preexposure phase of an LI study (e.g., Channell \& Hall, 1983; Hall \& Channell, 1985; see Honey, Iordanova, \& Good, 2010, for a review). Measuring the response to the stimulus during preexposure not only allows for an assessment of habituation, but also, importantly, allows for an assessment of the potentially confounding effect of the UR to the conditioned stimulus (CS) during conditioning. If the preexposure treatment produces LTH to the target stimulus, then differences in acquisition between the preexposed and control groups may have little to do with degrading the associative process. We are not the first to have noted this potential confound (e.g., Lovibond, Preston, \& Mackintosh, 1984; Lubow \& Siebert, 1969), but it is often ignored in practice, and the UR to the CS is rarely measured.

A common practice in LI studies (e.g., Feldon \& Weiner, 1991; Joseph et al., 2000; Lubow, Schnur, \& Rifkin, 1976; Nofrey, Ben-Shahar, \& Brake, 2008; and see Lubow \& 
Weiner, 2010a) is to run both the preexposure and conditioning phases "off baseline"; that is, the response to the CS is measured in neither preexposure nor conditioning. The presence and extent of LI is then assessed in a subsequent test session under extinction. This off-baseline procedure produces two primary problems that often make it impossible to judge the full extent of LI, or even its presence. First, as we discussed above, it obscures possible effects mediated by LTH of the UR to the preexposed stimulus. Second, differences that emerge during the course of extinction testing, which allows new learning to occur, are not an unambiguous test of LI during acquisition.

In the present experiments, we addressed both of these problems by using an on-baseline conditioned-suppression procedure to measure the response to repeated presentations of a white noise stimulus during the preexposure phase. Following the preexposure/habituation series, we looked for evidence of LI during one-trial-per-day acquisition of conditioned suppression, again on-baseline-a procedure that allowed us to assess the UR to the CS on the first trial for both nonpreexposed and preexposed animals. In addition, the onetrial-per-day procedure prevented the loss of the baseline behavior due to repeated shock presentations, and thus provided a detailed look at the development of LI in acquisition. LI should take the form of slowed acquisition of conditioned suppression following stimulus preexposure. However, any differences that occur on the first trial of conditioning, prior to reinforcement being presented, must be the result of LTH of unconditioned suppression in the preexposed group and the absence of habituation in the nonpreexposed controls.

Following conditioning to asymptote, the possible effects of preexposure on the course of subsequent extinction were examined in daily sessions. Do the effects of preexposure survive conditioning to asymptote, and if so, what impact do they have on the new learning in extinction? Westbrook and Bouton (2010) maintained that the associations formed during preexposure are not erased by conditioning, a position supported by data showing the recovery of LI with a context change (Westbrook, Jones, Bailey, \& Harris, 2000). However, the impact of preexposure on extinction is not clear. Tests in extinction that follows conditioning are used to assess LI in off-baseline procedures, but following the course of extinction is not a routine feature of LI experiments. A few experiments have reported the course of extinction after LI (e.g., Leung \& Westbrook, 2010; Lovibond et al., 1984; Lubow, Markman, \& Allen, 1968; Rosenberg, Holmes, Harris, \& Westbrook, 2011; Westbrook et al., 2000), and these have consistently shown either no difference in extinction or faster extinction to a preexposed CS. The conditions of these experiments have varied widely. The extent of LI in acquisition is not always clear (e.g., Lovibond et al., 1984; Rosenberg et al., 2011), and, importantly for studies of extinction, it is often difficult to know whether the groups are trained to a common asymptote (Leung \& Westbrook, 2010; Westbrook et al., 2000). Through the present experiments, we tried to resolve some of these ambiguities.

\section{Experiment 1}

In Experiment 1, we used a lick-suppression procedure to measure, during preexposure, the response to repeated presentations of a white noise stimulus. Following the preexposure/ habituation series, we looked for evidence of LI in a one-trialper-day conditioned lick-suppression procedure. Following conditioning to asymptote, we went directly to extinction to examine any enduring effects of preexposure that might be revealed in the course of extinction over days.

\section{Method}

Subjects Twenty-eight experimentally naïve male SpragueDawley rats (Charles River), 78 days old at the start of the experiment, were housed in pairs in plastic cages $(22 \times 42 \times$ $20 \mathrm{~cm}$ ) in a colony room maintained on a 12:12-h light:dark cycle. Experimentation took place during the light period of the cycle. Animals had ad lib access to food and water for three weeks after arrival before behavioral testing began. The experiment was approved by the Institute Animal Care and Use Committee of St. Mary's College of Maryland, and the procedures were consistent with the ethical guidelines of the American Psychological Association.

Apparatus The behavior procedures were carried out in four identical operant chambers $(30 \times 24 \times 21 \mathrm{~cm}$; Med Associates) enclosed within sound-attenuating chambers (61 $\times 45 \times 63 \mathrm{~cm}$ ) with background noise ( $66 \mathrm{~dB} \mathrm{SPL})$ provided by an exhaust fan. The two facing walls and the top were clear plastic. The other walls were aluminum. The grid floor was stainless steel rods (5-mm diameter) spaced $1.5 \mathrm{~cm}$ apart (center to center). A photo-beam lick system (Med Associates ENV-251L) was centered on one wall with a white cue light ( $28 \mathrm{~V}, 2.5-\mathrm{cm}$ white lens) located directly above. The 20 -s white noise stimulus (88-dB SPL, 10-ms rise-fall, with intensity measured with a Med Associates ANL-929A system) was generated by a Med Associates stimulus generator (ANL-926) and delivered through a high-frequency speaker (Med Associates ENV-225BW). Foot shocks were generated by a Med Associates unit (ENV-414s) for each chamber.

Procedure Rats were restricted to 60 min of water per day and given four days of preliminary lick training (20-min sessions) in operant chambers placed in an open room. This preliminary training was followed by seven daily 10-min sessions in the operant chambers used for testing. Two groups of rats were created, matched on their overall rates of licking. 
Minimum lick criteria were used for all trials in which suppression was measured. The first stimulus presentation of a session came no sooner than $60 \mathrm{~s}$ into the session and only after the animal had made 200 licks. The animal had to have made 50 licks in the last $20 \mathrm{~s}$ and five in the last $1 \mathrm{~s}$ prior to stimulus onset. These criteria were met on all first trials of each session for each rat. Licking remained strong throughout the experiment. The mean number of licks per 10-min session was 2,217 (range: 1,721-2,724). Although the total number of licks declined over conditioning sessions and then recovered during extinction, $F(6.70,174.29)=2.43, p<.05, \eta_{\mathrm{p}}{ }^{2}=.09$, $95 \%$ CI: $[.00, .14]$, a Group $\times$ Session ANOVA showed no significant group difference or interaction $(p \mathrm{~s}>.40)$.

Group PE $(n=14)$ received two 10 -min preexposure/ habituation sessions on consecutive days. Each session consisted of ten presentations of the white noise stimulus that would become the conditioned stimulus (CS) in the subsequent conditioning sessions. The first three stimuli each day were presented only when the rat met the consistent-licking criteria, with a minimum 60-s intertrial interval (ITI). The additional seven stimuli were presented on a fixed 40-s ITI without regard to behavior. The rats in Group NPE $(n=14)$ were placed in the test chambers and allowed to lick without any imposed stimuli.

The first of four daily 10-min conditioning sessions began $24 \mathrm{~h}$ after the last preexposure/habituation session. In each session, each animal received a single pairing of the CS with a $0.5-\mathrm{mA}, 0.5$-s foot shock while it was consistently licking. The CS duration was extended to $20.5 \mathrm{~s}$ so that a 20 -s sample of licking could be obtained before the onset of the foot-shock US. The CS and US coterminated.
Extinction sessions began $24 \mathrm{~h}$ after the last conditioning session. In each of six sessions, rats received three presentations of the 20-s CS following the consistent-licking criteria. No shocks were delivered.

Data analysis Standard lick suppression ratios were calculated as (licks during the 20-s CS) / (licks during 20-s PreCS + licks during the 20-s CS). Statistical analyses over trials or days were based on repeated measures analyses of variance (ANOVAs) with Group as a between-subjects factor. The significance level was set at $\alpha=.05$. When sphericity could not be assumed (Mauchly's test), the Greenhouse-Geisser correction was used. Individual trial comparisons across the two groups were based on two-tailed $t$ tests with $\alpha=.05$. When Levine's test for equality of variances was significant, the degrees of freedom were corrected.

\section{Results}

Preexposure/habituation The left panel of Fig. 1 shows licksuppression ratios for the first three presentations of the white noise stimulus during each session. Only animals in Group PE received stimulus presentations on these days. The licking behavior for Group NPE was measured on "blank trials," as though stimuli had been delivered, following the same timing and lick criteria that were used for the PE group.

The first stimulus presentation to animals in Group PE provoked strong unconditioned suppression of licking ( $M=$ .07). Robust long-term habituation occurred across days, as measured by responsiveness on the first trial of each of the two preexposure sessions and the first conditioning trial, which

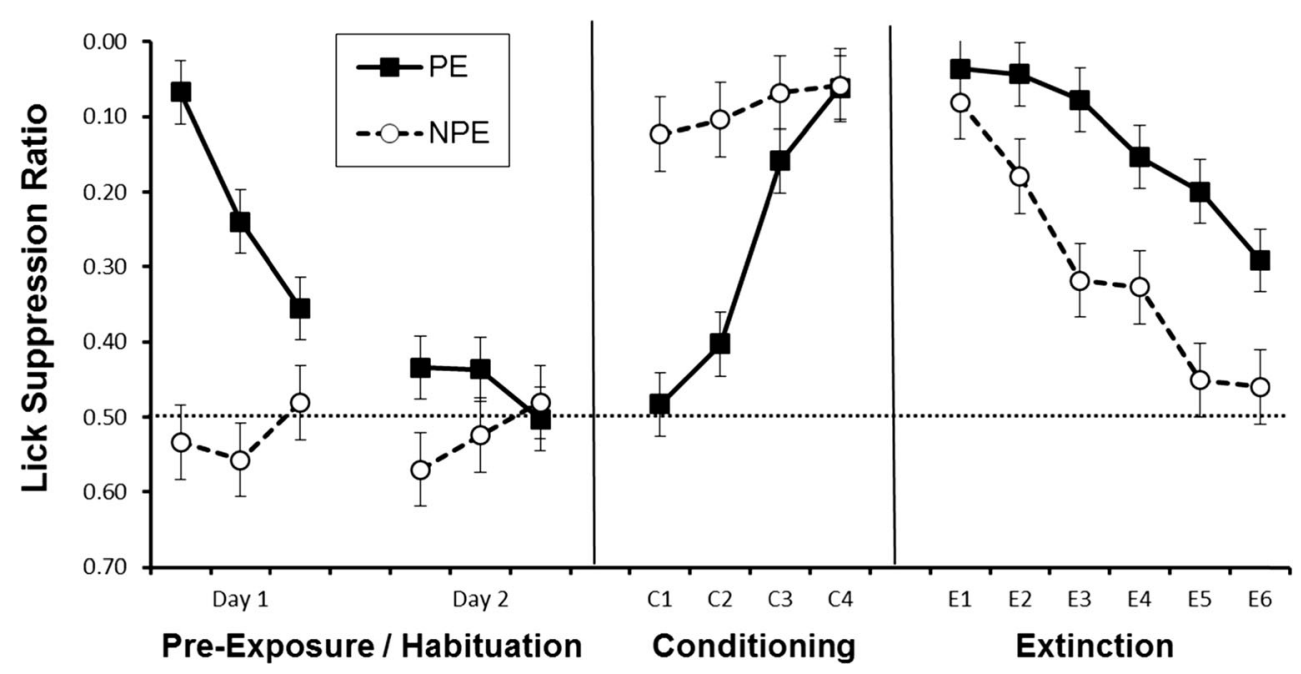

Fig. 1 Mean lick suppression ratios to the conditioned stimulus (CS) in Experiment 1 . The rats in Group PE had received ten CS presentations per day during the two preexposure/habituation sessions (left panel), whereas the rats in Group NPE had licked without any CS presentations. Only the first three CS presentations each day are shown. Both groups received one
CS paired with a foot-shock unconditioned stimulus each day during conditioning (center panel). Both groups then received three CS presentations per day during extinction (right panel). Only the first trial of each extinction day is shown. The vertical bars represent SEMs. 
occurred before the first foot shock. A one-factor repeated measure ANOVA across these first trials was significant, $F(1.39$, $18.13)=68.15, p<.001, \eta_{\mathrm{p}}{ }^{2}=.84,95 \%$ confidence interval (CI): $[.63, .90]$. Baseline licking during the PreCS periods before each CS presentation was robust for both groups (Group PE, $M=99.1$; Group NPE, $M=103.8$ ). A Group $\times$ Day ANOVA on the PreCS data produced no significant results ( $p s>.31$ ).

Short-term habituation of lick suppression was observed over the first three measured trials within days for Group PE. A Day $\times$ Trial repeated measures ANOVA showed significant decreases across days, $F(1,11)=64.42, p<.001, \eta_{\mathrm{p}}{ }^{2}=$ $.85,95 \%$ CI: $[.55, .91]$, and trials, $F(2,22)=11.56, \eta_{\mathrm{p}}{ }^{2}=.51$, $95 \%$ CI: $[.16, .67]$. The Day $\times$ Trial interaction was significant, since there was a much larger within-session decrease in responsiveness on Day 1 than on Day 2, $F(2,22)=7.50, p<$ $.01, \eta_{\mathrm{p}}{ }^{2}=.41,95 \% \mathrm{CI}$ : $[.07, .59]$. (Two rats failed to lick during the PreCS and CS periods on the third trial of Day 2. The data from these two animals were excluded from the analysis of short-term habituation.)

Conditioning Lick-suppression ratios for the one-trial-perday conditioning phase are presented in the middle panel of Fig. 1. The animals in Group NPE, which heard the CS for the first time on the first conditioning trial, showed strong unconditioned suppression to the CS $(M=.12)$, whereas the animals in Group PE remained habituated to the CS $(M=.48)$. This group difference was significant, $t(26)=10.56, p<.001, \eta_{\mathrm{p}}{ }^{2}=$ $.81,95 \%$ CI: $[.64, .87]$

As is shown in Fig. 1, Group PE reached the response asymptote more slowly than did Group NPE, resulting in an overall group difference across the four trials, $F(1,26)=$ 66.35, $p<.001, \eta_{\mathrm{p}}{ }^{2}=.72,95 \%$ CI: [.49, .81]. The day main effect was also significant, $F(2.39,62.02)=39.04, p<$ $.001, \eta_{\mathrm{p}}{ }^{2}=.60,95 \%$ CI: [.43, .69], as was the Group $\times$ Day interaction, $F(2.39,62.02)=21.07, p<.001, \eta_{\mathrm{p}}{ }^{2}=.45$, $95 \%$ CI: $[.25, .57]$. This pattern of results suggests that LI was observed, but that conclusion is confounded by the significant first-day $(\mathrm{C} 1)$ difference. Post-hoc $t$ tests comparing the groups on each day produced significant differences indicative of LI on $\mathrm{C} 2, t(17.97)=6.30, p<.001, \eta_{\mathrm{p}}{ }^{2}=.69$, $95 \%$ CI: [.37, .81], but not on C3, $t(13.81)=1.82, p=.080$, $\eta_{\mathrm{p}}{ }^{2}=.19,95 \%$ CI: [.00, .49], or C4, $t(26)=0.21, p=.835$, $\eta_{\mathrm{p}}{ }^{2}=.002,95 \%$ CI: $[.00, .06]$. Thus, statistical evidence for LI was found only on $\mathrm{C} 2$ following a single CS/US pairing the day before; unconditioned responding in Group NPE may still have contributed to the $\mathrm{C} 2$ group difference.

Baseline licking during the PreCS periods before each CS declined equally for the two groups across the four conditioning sessions, from a daily mean of 97.6 on Day $\mathrm{C} 1$ to 81.7 on Day C4. A Group $\times$ Day ANOVA produced a significant day main effect, $F(3,78)=4.222, p=.008, \eta_{\mathrm{p}}{ }^{2}=.14,95 \% \mathrm{CI}$ : $[.01, .26]$, but neither the group main effect, $F(1,26)=1.386$, $p=.25, \eta_{\mathrm{p}}{ }^{2}=.05,95 \%$ CI: $[.00, .26]$, nor the Group $\times$ Day interaction, $F(3,78)=1.890, p=.14, \eta_{\mathrm{p}}{ }^{2}=.07,95 \%$ CI: [.00, .17], was significant.

Extinction Suppression ratios for the first trial of each extinction session are shown in the right panel of Fig. 1. Both groups began extinction with suppression ratios near zero, suggesting that they had reached a common asymptote, and we found no significant difference between groups on the first extinction session $(\mathrm{E} 1), t(14.81)=1.753, p=.10$. Both groups exhibited significant extinction over the six extinction sessions, but Group PE extinguished significantly more slowly than did Group NPE. This slowed extinction for Group PE was reflected in both a significant group main effect, $F(1,26)=$ $21.59, p<.001, \eta_{\mathrm{p}}{ }^{2}=.45,95 \% \mathrm{CI}$ : [.16, .63], and a significant Group $\times$ Day interaction, $F(5,130)=2.55, p=.031, \eta_{\mathrm{p}}{ }^{2}=.09$, $95 \%$ CI: $[.00, .16]$. The day main effect also was significant, $F(5,130)=26.50, p<.001, \eta_{\mathrm{p}}{ }^{2}=.50,95 \%$ CI: $[.37, .58]$. Post-hoc $t$ tests showed Group PE to be significantly more suppressed than Group NPE on each trial except E1 ( $p$ s $<.04)$.

Baseline licking remained stable across the six extinction sessions (overall mean $=93.8$ ), since no term in a Group $\times$ Day ANOVA of PreCS licking was significant ( $p \mathrm{~s}>.17)$.

\section{Discussion}

The basic habituation and LI data are not surprising, but two elements of the results are worthy of note. First, a cautionary note: If the UR to the preexposed stimulus is not measured, and that preexposure produces LTH, much of what is considered LI may result from the difference between the preexposed and nonpreexposed animals in their URs to the initial presentations of the CS during conditioning. Had we run preexposure and acquisition off-baseline, we would have been blind to the significant difference between the groups on the first conditioning trial, which was due solely to the UR to the CS in the nonpreexposed group and the LTH of that response in the preexposed group. Had we run a two- or three-trial acquisition session off-baseline and then looked for LI in a subsequent test session, the group difference presumed to represent LI would be largely the result of the initial UR difference. All off-baseline LI procedures make interpretation of the extent of LI difficult (e.g., Feldon \& Weiner, 1991; Lubow et al., 1976), and in some cases may lead to the potentially false conclusion that LI was found (e.g., Almey, Hafez, Hantson, \& Brake, 2013; Barak \& Weiner, 2007; Nofrey et al., 2008). These problems in interpretation may be particularly acute in experiments that use conditioned suppression designs or that assess the possible LI modulating effects of drug or brain manipulations (Feldon \& Weiner, 1991; Joseph et al., 2000; Peters \& Joseph, 1993; Tai, Cassaday, Feldon, \& Rawlins, 1995). In the typical off-baseline conditioned suppression design, it is impossible to know whether the brain or drug manipulation has affected LI, or disrupted habituation to 
the preexposed stimulus, or distorted the basic response to that stimulus.

Second, the slower extinction in the preexposed animals after both groups had been conditioned to a common asymptote was unexpected and potentially of theoretical importance. As we noted earlier, the relatively few LI experiments that have reported the course of extinction after LI (e.g., Leung \& Westbrook, 2010; Lovibond, et al., 1984; Lubow, et al., 1968; Rosenberg et al., 2011; Westbrook et al., 2000) have consistently found no extinction differences, or faster extinction in the preexposed group or to the preexposed CS.

\section{Experiment 2}

The robust and unexpected extinction effect found here is contrary to the published literature and demanded replication. Experiment 2 was an attempt at replication performed in a different laboratory with different experimenters using a slightly modified procedure and a different response measure.

\section{Method}

Subjects Sixteen male Long Evans rats (Harlan Laboratories, Indianapolis, IN), approximately 80 days old at the start of the experiment, were individually housed in a colony room maintained on a 14:10-h light:dark cycle. Experimentation took place during the light period of the cycle. The rats were food deprived to $85 \%$ of their initial body weights throughout the experiment. They were monitored and cared for in compliance with the Association for Assessment and Accreditation of Laboratory Care guidelines and the IACUC of Dartmouth College. The rats had performed in a previous experiment, in two different contexts in which they experienced shock. The contexts differed from the present contexts in terms of visual, tactile, and olfactory cues. The rats had never been exposed to auditory stimuli prior to the present experiment.

Apparatus The behavioral procedures were carried out in eight identical standard conditioning chambers $(24 \times 30.5 \times$ $29 \mathrm{~cm}$; Med Associates) enclosed in sound-attenuating chambers $(62 \times 56 \times 56 \mathrm{~cm})$ with background noise $(68 \mathrm{~dB}$ SPL $)$ provided by an exhaust fan. The chambers (Med Associates, ENV-007) consisted of aluminum front and back walls and clear acrylic sides and top. The grid floor was stainless steel rods (5-mm diameter) spaced $1.5 \mathrm{~cm}$ apart (center to center). Each chamber was outfitted with a food cup, recessed in the center of the front wall. Retractable levers (Med Associates, model ENV-112CM) were positioned to the left and right of the food cup. (The right lever remained retracted during the experiment.) The chambers were illuminated by one $6-\mathrm{W}$ bulb, with a red cover, mounted to the ceiling of the soundattenuating chamber. The auditory stimulus was $30 \mathrm{~s}$ of white noise (88-dB SPL, 10-ms rise-fall, with intensity measured with an EXTECH Instruments sound level meter, A scale) produced by a Med Associates stimulus generator (ANL926), delivered through a speaker mounted $15 \mathrm{~cm}$ above and to the right of the food cup. The apparatus was controlled by computer equipment located in an adjacent room. The reinforcer was a 45-mg grain-based rodent food pellet (Bioserv). Foot shocks were generated by a Med Associates unit (ENV414) for each chamber.

Procedure On the first day of the experiment, each rat was assigned to one chamber and received a single 1-h session of magazine training. Food pellets were delivered freely on a random-time 60-s (RT 60-s) schedule, resulting in approximately 60 pellets being delivered. In addition, all lever presses were reinforced with a single pellet.

On the following two days, rats received a 1-h session in which lever responding was reinforced on a random-interval (RI) 15-s schedule. On the next day, the schedule was increased to RI $30 \mathrm{~s}$ for two days, and for the ten subsequent days the schedule was RI $60 \mathrm{~s}$.

Three days of preexposure/habituation followed baseline training. Rats received daily 1 -h sessions in which lever pressing was reinforced on a RI 60-s schedule. The rats in Group PE $(n=8)$ received ten 30 -s presentations of the CS in each session, for a total of 30 preexposure presentations. The intertrial interval (stimulus offset to stimulus onset) was variable $(M=5.15 \mathrm{~min} \pm 25 \%)$. For the other half of the rats (Group NPE, $n=8$ ), no stimuli were presented.

For conditioning, all rats remained on the RI 60-s schedule during four daily 1 -h sessions. At Minute 29 of each session, the CS was presented; upon its termination, a $0.5-\mathrm{mA}, 0.5-\mathrm{s}$ foot shock was delivered. In this way, each rat received four conditioning trials, one per session for four daily sessions.

The extinction phase was identical to the conditioning phase, with the exception that the foot shock was never delivered. After 14 initial daily one-trial sessions, ten additional daily extinction sessions were presented, with three trials per session. The first trial occurred $29 \mathrm{~min}$ into the session, and the next two trials occurred at subsequent 10-min intervals.

Data analysis Standard suppression ratios were calculated as (lever presses during the 30-s CS) / (lever presses during the 30 -s PreCS period + lever presses during the 30 -s CS). The data were analyzed with the same statistical procedures used in Experiment 1.

Results

Preexposure/habituation Figure 2 shows suppression ratios during the preexposure/habituation phase. Responding for Group NPE was calculated during "blank trials" over the identical time periods that the CS was present for Group PE. 


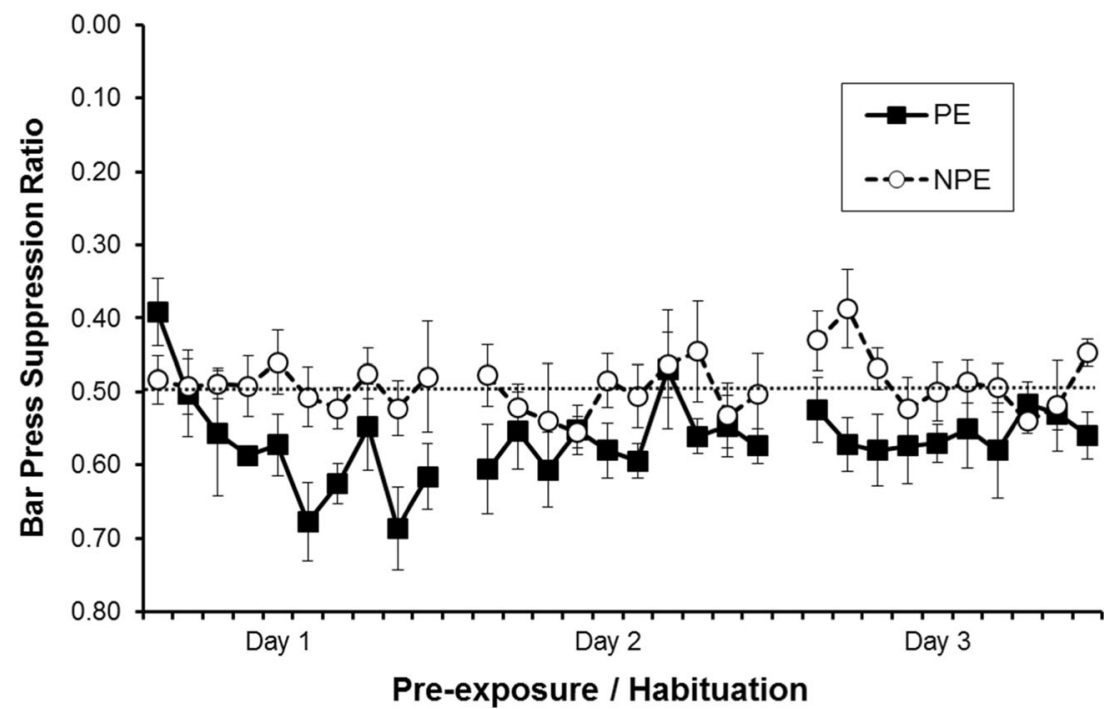

Fig. 2 Mean bar-press suppression ratios to the conditioned stimulus (CS) during preexposure in Experiment 2. The rats in Group PE received ten $\mathrm{CS}$ presentations per day during the three sessions, whereas the rats in Group NPE pressed without any CS presentations. The vertical bars represent $S E M \mathrm{~s}$

slight increase in baseline lever pressing for both groups across days. A Group $\times$ Days $\times$ Trials ANOVA of PreCS lever pressing produced a main effect of day, $F(2,28)=3.44, p=$ $.046, \eta_{\mathrm{p}}{ }^{2}=.20,95 \%$ CI: $[.00, .40]$. No other main effects or interactions were significant, including all group-related terms $(p s>.71)$.

Conditioning Suppression ratios for the one-trial-per-day conditioning phase are presented in the left panel of Fig. 3. Groups NPE and PE were not significantly different on C1, $F(1,14)=1.23, p=.29$. Thus, in contrast to Experiment 1, we did not observe substantial unconditioned suppression when Group NPE heard the CS for the first time on C1. This is did not differ between the groups. There was, however, a

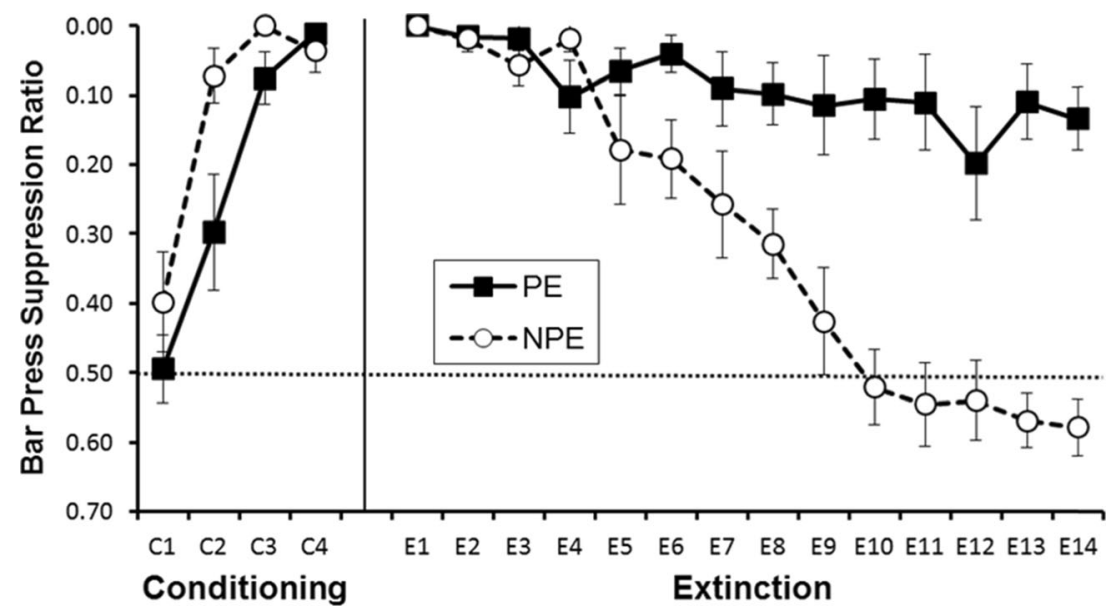

Fig. 3 Mean bar-press suppression ratios to the conditioned stimulus (CS) during conditioning and extinction in Experiment 2. The rats in Group PE had been preexposed to the CS during the preexposure/ habituation phase of the experiment, whereas the rats in Group NPE had been placed in the test chamber without CS presentations. The rats in both groups received one CS paired with a foot-shock unconditioned stimulus each day during conditioning (left panel). The rats in both groups then received one CS presentation per day during extinction (right panel). The vertical bars represent SEMs 
consistent with the lack of significant unconditioned suppression in Group PE during preexposure (Fig. 2). Inspection of Fig. 3 suggests that LI was observed in Group PE, since these animals were slower to suppress bar pressing over days, resulting in an overall group difference across the four conditioning sessions, $F(1,14)=5.82, p=.03, \eta_{\mathrm{p}}{ }^{2}=.30,95 \% \mathrm{CI}$ : $[.00, .56]$. The day main effect was also significant, $F(1.9$, $26.6)=37.72, p<.001, \eta_{\mathrm{p}}{ }^{2}=.72,95 \% \mathrm{CI}:[.49, .81]$, but the Group $\times$ Day interaction was not, $F(1.9,26.6)=2.52, p=$ $.10, \eta_{\mathrm{p}}{ }^{2}=.15,95 \% \mathrm{CI}:[.00, .36]$. Post-hoc comparisons on each day were run to verify the impression from Fig. 3 that LI emerged on $\mathrm{C} 2$ as a result of the first conditioning trial the day before. Group NPE had significantly stronger suppression on $\mathrm{C} 2, t(10)=2.45, p<.03, \eta_{\mathrm{p}}{ }^{2}=.30,95 \% \mathrm{CI}:[.00, .57]$, and marginally more suppression on $\mathrm{C} 3, t(7)=2.02, p=.08, \eta_{\mathrm{p}}{ }^{2}=$ $.23,95 \%$ CI: $[.00, .21]$. The groups did not differ significantly on $\mathrm{C} 4, t<1$. Thus, LI was present on $\mathrm{C} 2$ as a result of the first CS-US pairing the day before, but it was gone by $\mathrm{C} 4$ as the animals in Group PE became conditioned to asymptote. This pattern of differences was sufficient to produce the significant group main effect from the ANOVA across the four days.

An analysis of PreCS lever pressing during the conditioning phase did not reveal any significant main effects or interactions. Over the course of conditioning, during the PreCS period, the lever-pressing means were identical for Groups PE and NPE, at 6.41.

Extinction The suppression ratios for the first 14 daily trials of extinction are presented in the right portion of Fig. 3. Both groups suppressed their lever pressing completely on E1, indicating that conditioning had resulted in asymptotic suppression. Consistent with the results of Experiment 1, extinction was delayed for Group PE relative to Group NPE. An ANOVA over the 14 days revealed main effects of group, $F(1$, $14)=30.78, p<.001, \eta_{\mathrm{p}}{ }^{2}=.69,95 \% \mathrm{CI}:[.31, .81]$, and day, $F(4.30,60.2)=18.35, p<.001, \eta_{\mathrm{p}}{ }^{2}=.57,95 \% \mathrm{CI}:[.34, .65]$, and an interaction between group and day, $F(4.3,60.2)=8.71$, $p<.001, \eta_{\mathrm{p}}{ }^{2}=.38,95 \% \mathrm{CI}:[.14, .49]$. We observed no significant change in the suppression ratios for Group PE over the 14 trials, $F(13,91)=1.64, p=.087$, although there was a robust decline for Group NPE, $F(13,91)=22.68, p<.001, \eta_{\mathrm{p}}{ }^{2}$ $=.75,95 \%$ CI: $[.64, .79]$.

No significant differences in PreCS lever pressing emerged over the course of the first 14 daily trials of extinction. Lever pressing during the PreCS period averaged 6.96 and 6.67 for Groups PE and NPE, respectively.

After 14 extinction sessions with a single CS presentation per day, the suppression of bar pressing for Group PE remained near the conditioning asymptote. The number of CS presentations was increased to three per day for an additional ten extinction sessions (Fig. 4). Group PE continued to show slower between-session extinction than Group NPE, but suppression was mostly extinguished by the final session of extinction. Furthermore, within each session there was a general pattern for Group PE to show relatively high levels of suppression on the first trial, followed by within-session (short-term) extinction. In contrast, Group NPE showed low levels of suppression over this entire phase. An ANOVA revealed main effects of day, $F(9,126)=4.26, p<.001, \eta_{\mathrm{p}}{ }^{2}=$ $.23,95 \%$ CI: $[.07, .30]$, and group, $F(1,14)=9.70, p<.008$, $\eta_{\mathrm{p}}{ }^{2}=.41,95 \% \mathrm{CI}:[.04, .64]$. We also found significant interactions between day and group, $F(9,126)=3.68, p<.001$,

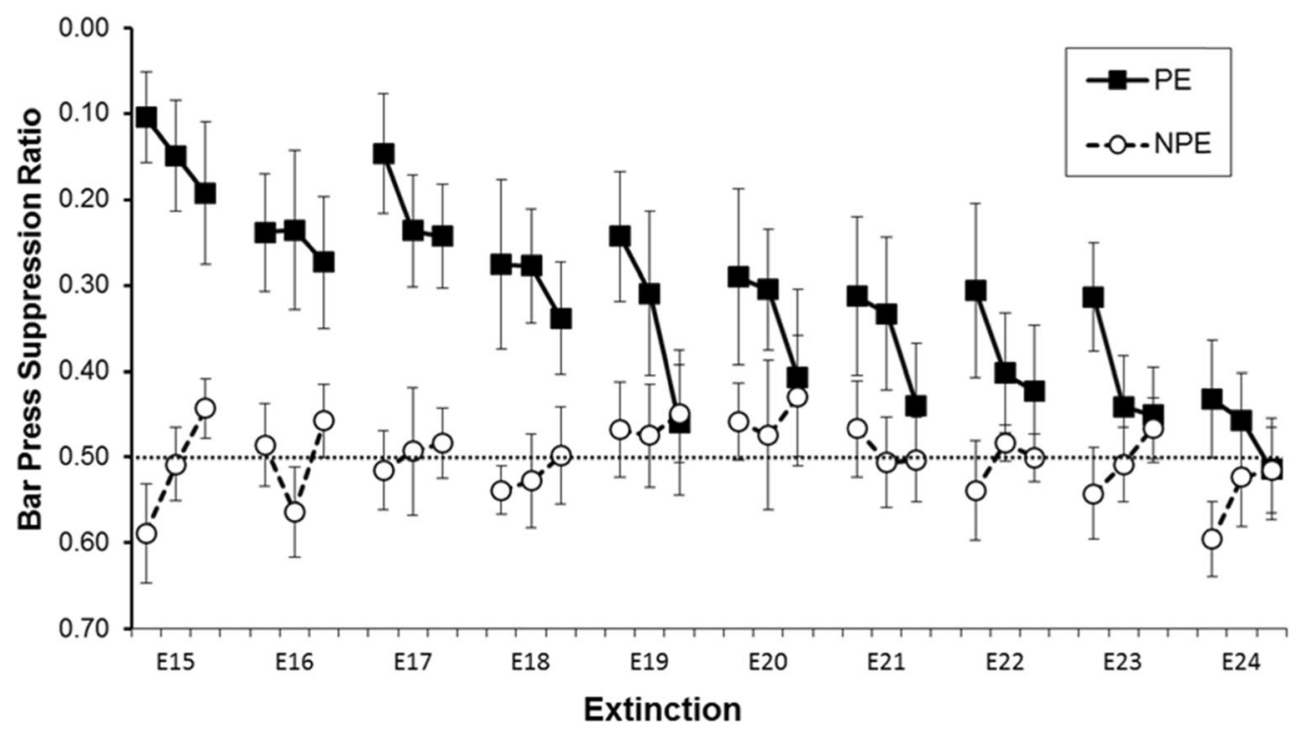

Fig. 4 Mean bar-press suppression ratios to the conditioned stimulus (CS) during the final extinction sessions in Experiment 2. The rats in Group PE had been preexposed to the CS during the preexposure/ habituation phase of the experiment, whereas the rats in Group NPE had been placed in the test chamber without CS presentations. Following conditioning and initial extinction (Fig. 3), the rats in both groups received three CS presentations per day for a final ten days. The vertical bars represent $S E M \mathrm{~s}$ 
$\eta_{\mathrm{p}}{ }^{2}=.21,95 \%$ CI: $[.05, .28]$, as well as trial and group, $F(2,28)=12.52, p<.001, \eta_{\mathrm{p}}{ }^{2}=.47,95 \%$ CI: $[.16, .63]$.

During these final 10 days of extinction, there were no significant differences in PreCS responding. PreCS responding averaged 7.91 and 8.23 for Groups PE and NPE, respectively.

\section{Discussion}

The main result of Experiment 2 was the significant replication of the delayed extinction effect in the preexposed group in Experiment 1. This replication was performed with a different response measure, showing that the extinction effect is not related to some idiosyncrasy of a lick suppression procedure. In addition, the bar-press suppression measured in Experiment 2, in contrast to the lick suppression of Experiment 1, showed little initial unconditioned suppression to stimulus presentations during preexposure, and no measurable habituation over the course of preexposure. Thus, these response characteristics are not essential for the extinction effect. The significant group difference in suppression ratios during the preexposure sessions, produced by unexpected CS-facilitated responding in the PE group, was reduced over the last few trials, but this difference could have made some contribution to the development of LI. Importantly, the PE group's suppression ratio on the first conditioning trial was near .50 , and the group difference on the first conditioning trial was not significant. Furthermore, we found no significant group differences in baseline responding throughout conditioning or extinction.

\section{General discussion}

In two experiments based on a conditioned suppression LI design with rats, we measured (1) the initial response and habituation to the target stimulus during the preexposure phase, (2) LI in trial-by-trial acquisition in a one-trial-perday conditioning phase, and (3) the course of extinction over days following the conditioning of preexposed and control animals to a common asymptote. The results emphasize the importance of measuring the UR to the target stimulus and measuring LI during conditioning. The data for the course of extinction, only rarely considered in LI experiments, yielded a result that was neither empirically nor theoretically expected: The preexposed animals were significantly more resistant to extinction than the controls.

In measuring habituation to the target stimulus during preexposure, we were able to assess the influence of the UR to the CS during conditioning. In Experiment 1, we observed significant initial responses to the stimulus during preexposure that showed LTH over days. This behavioral pattern led to a significant group difference on the first conditioning trial, due to the UR to the CS in the NPE group, a UR that had habituated in the PE group. The procedures of Experiment 2, on the other hand, yielded little initial response to the target stimulus and no measureable STH or LTH. Many differences between these two experiments could account for the measured habituation differences, but the UR to the CS did not confound the first conditioning trial in Experiment 2. We are not the first to have pointed out the possible confounding effect of the UR to the CS in LI experiments (e.g., Lovibond et al., 1984; Lubow $\&$ Siebert, 1969), but the problem is often ignored in practice (Feldon \& Weiner, 1991; Joseph et al., 2000; Lubow et al., 1976; Peters \& Joseph, 1993; Tai et al., 1995). Unless one measures the UR to the CS, it cannot be known whether the UR has had little effect on the measurement of LI, as in Experiment 2, or a large and significant effect, as in Experiment 1.

The importance of measuring conditioning trial by trial was emphasized in both experiments, in that each showed a very narrow window during conditioning for the demonstration of LI. In individual-trial comparisons, only the second conditioning trial in each experiment showed evidence of significant LI. If the UR to the target stimulus during preexposure - or at least the response, preshock, to the CS on the first conditioning trial-is not measured, the extent of LI in this narrow window is unknown. Given the narrowness of this window, its likely dependence on the CS and US parameters, and the ambiguity created by the UR to the CS, the only unambiguous index of LI is trial-by-trial conditioning. In off-baseline procedures that measure LI in test sessions following blind preexposure and conditioning, LI depends critically on the number of conditioning trials: Too many may drive all groups to asymptote and obscure LI; too few, and LI may be an artifact of UR differences.

Even our conditioning data in Experiment 1 are somewhat ambiguous. We do not know how much of the Trial 2 difference was related to the UR difference in Trial 1. One solution to this problem would be to use target stimuli and response measures that do not produce initial UR differences in conditioning. That was true of the particular parameters and measures of bar-press suppression used in Experiment 2, but significant STH and LTH have been shown for bar-press suppression in other experiments (e.g., Jordan, Strasser, \& McHale, 2000; Leaton \& Borszcz, 1990). Alternatively, the UR to the CS in the NPE group could be reduced by limited stimulus presentations during preexposure. Significant LTH can be produced with one or two stimulus presentations (e.g., Leaton, 1976), but there has been no report of LI being produced by such limited preexposure. These problems may also be minimized in designs that use measures other than response suppression or in which the conditioned response is different from the UR to the CS. In any case, the confounding effect of the UR can only be known if the UR to the CS is measured. 
The significantly delayed extinction shown by the preexposed groups in both experiments was unexpected. The robust nature of the effect in Experiment 1 was shown by the replication in Experiment 2, in a different laboratory and with a different response measure. Although LI is commonly measured under extinction conditions in test sessions following conditioning, the focus is on degraded acquisition, and no attempt is made to condition the animals to a common asymptote (e.g., Westbrook et al., 2000). Very few studies have followed the course of extinction over trials or days, and those that have, have consistently shown either no extinction difference or faster extinction in the preexposed animals or to the preexposed stimuli. Lubow et al. (1968), conditioning the pinna response in rabbits to asymptote, found no preexposure extinction difference over 160 trials (Exp. 1), or faster extinction in the preexposed group over 20 trials (Exp. 2). Leung and Westbrook (2010) found consistently lower rates of freezing to preexposed stimuli over as many as 24 extinction trials, but the conditioning phase did not achieve asymptotic response levels. In a conditioned suppression design, Lovibond et al. (1984) found no difference between preexposed and control rats in five one-trial-per-day extinction trials, but again, it is not clear that the groups were conditioned to a common asymptote. In an exception to this pattern of results, Vervliet (2013) found mixed results in a human shock expectancy experiment. Over 12 extinction trials, the preexposed subjects extinguished faster on early trials but failed to achieve the same final level of extinction as controls.

We have emphasized conditioning to a "common asymptote" throughout this discussion. It is impossible to interpret differences in extinction unless the groups begin extinction at a common level. Of course, a response asymptote may not reflect an asymptote in the underlying associative strength. A suppression ratio like that used in the present experiments may reach a response asymptote at or near zero while associative strength continues to increase. If our response measure had suffered from a ceiling effect, we believe it would have worked against the observed extinction effect. Since acquisition was retarded in the PE groups, we assume that it would have been the NPE groups that hypothetically began extinction at a higher level of associative strength. If this were true, it should have been the NPE groups that showed higher response levels in extinction. We found the opposite.

Perhaps the most relevant experiment for comparison with the present data is Rosenberg et al. (2011). In a within-subjects conditioned freezing paradigm, they reported an extensive analysis of extinction. After 120 trials of preexposure over six days, they conditioned both preexposed and control stimuli to a common asymptote. They then ran ten extinction trials a day for four days, and in extinction found significantly less freezing to the preexposed stimulus. This pattern of results was consistent over four separate experiments and appears to be in direct opposition to the pattern of results reported here. Many differences between the Rosenberg et al. (2011) experiment and the present experiments could have been important in generating the opposite outcomes in extinction. A particularly important difference may have been their use of a within-subjects procedure, as compared with our between-subjects procedure. We know that contextual associations are important for understanding LI (Hall \& Channell, 1985; Hall \& Honey, 1989; Lovibond et al., 1984; Westbrook et al., 2000), and that between- versus within-subjects procedural differences may produce important differences in contextual associations. There is evidence that another significant behavioral phenomenon, the partial reinforcement extinction effect (PREE), changes with this variable. The PREE is reliably found in between-group designs, but not in within-subjects designs, and this difference may depend on contextual associations (Papini, Thomas, \& McVicar, 2002). These considerations suggest that a context manipulation may shed light on our delayed extinction effect and the differences between our results and those previously reported.

Although there is no generally accepted theory of LI (Lubow \& Weiner, 2010a), all theories have focused on the relation between preexposure and the acquisition or expression of conditioning, and few have been extended to explain extinction following asymptotic conditioning. Lubow and Weiner (2010a) suggested that LI theories fall into two broad general categories. The first category includes attention-like theories that account for LI by decreases in the associability of the stimuli during preexposure. Such theories would have trouble with our extinction data unless the change in associability remained depressed through asymptotic conditioning. To the best of our knowledge, no theory makes that assumption. The second broad category of theories accounts for LI through competition among memorial processes that typically depend on contextual associations. For example, the CS-nothing memory from preexposure is contextdependent and interferes with the acquisition or expression of a CS-US memory. Westbrook and Bouton's (2010) theoretical perspective falls within this category of LI theories, and they are clear in their assumption that the effects of preexposure survive conditioning to asymptote. Their explicit prediction is that extinction should be faster in preexposed animals, because the conditions of extinction restore the CSnothing memory formed during preexposure. In our hands, preexposure interfered with the development of extinction. It does seem clear that no current theory of LI easily accommodates the robust extinction effect shown in the present experiments, and since the results were unexpected, we have no readily available theoretical explanation. Our speculations lead us toward memorial processes that are dependent on contextual associations, especially considering that both LI and extinction are known to depend on contextual associations. 
The present experiments leave us with two important conclusions. First, the procedures used in many experiments may confound the interpretation of LI. In some cases, neither the extent nor even the presence of LI can be unambiguously assumed. Second, extinction following conditioning to asymptote can be significantly prolonged in animals preexposed to the CS, as compared to animals conditioned without preexposure. This finding was empirically and theoretically unexpected and may provide new insights for theories of LI.

Author note We thank Walter Bowles for help with the data collection for Experiment 1. The research done at St. Mary's College was supported in part by faculty development grants to W.P.J. The research done at Dartmouth College was supported in part by grants from the National Institute of Mental Health (No. F32MH092991) to T.P.T. and the National Institutes of Health (No. R01 DA027688) to D.J.B.

\section{References}

Almey, A., Hafez, N. M., Hantson, A., \& Brake, W. G. (2013). Deficits in latent inhibition induced by estradiol replacement are ameliorated by haloperidol treatment. Frontiers in Behavioral Neuroscience, 7, 136. doi:10.3389/fnbeh.2013.00136

Barak, S., \& Weiner, I. (2007). Scopolamine induces disruption of latent inhibition which is prevented by antipsychotic drugs and an acetylcholinesterase inhibitor. Neuropharmacology, 32, 989-999.

Channell, S., \& Hall, G. (1983). Contextual effects in latent inhibition with an appetitive conditioning procedure. Animal Learning \& Behavior, 11, 67-74. doi:10.3758/BF03212309

Feldon, J., \& Weiner, I. (1991). The latent inhibition model of schizophrenia attention disorder: Haloperidol and sulpiride enhance rats' ability to ignore irrelevant stimuli. Biological Psychiatry, 29, 635646.

Hall, G., \& Channell, S. (1985). Differential effects of contextual change on latent inhibition and on the habituation of an orienting response. Journal of Experimental Psychology: Animal Behavior Processes, 11, 470-481. doi:10.1037/0097-7403.11.3.470

Hall, G., \& Honey, R. C. (1989). Contextual effects in conditioning, latent inhibition, and habituation: Associative and retrieval functions of contextual cues. Journal of Experimental Psychology: Animal Behavior Processes, 15, 232-241. doi:10.1037/0097-7403.15.3.232

Honey, R. C., Iordanova, M. D., \& Good, M. (2010). Latent inhibition and habituation: Evaluation of an associative analysis. In R. Lubow \& I. Weiner (Eds.), Latent inhibition: Cognition, neuroscience, and applications to schizophrenia (pp. 163-179). New York, NY: Cambridge University Press.

Jordan, W. P., Strasser, H. C., \& McHale, L. (2000). Contextual control of long-term habituation in rats. Journal of Experimental Psychology: Animal Behavior Processes, 26, 323-339. doi:10.1037/0097-7403. 26.3.323

Joseph, M. H., Peters, S. L., Moran, P. M., Grigoryan, G. A., Young, A. M. J., \& Gray, J. A. (2000). Modulation of latent inhibition in the rat by altered dopamine transmission in the nucleus accumbens at the time of conditioning. Neuroscience, 101, 921-930.

Leaton, R. N. (1976). Long-term retention of the habituation of lick suppression and startle response produced by a single auditory stimulus. Journal of Experimental Psychology: Animal Behavior Processes, 2, 248-259. doi:10.1037/0097-7403.2.3.248
Leaton, R. L., \& Borszcz, G. S. (1990). Hippocampal lesions and temporally chained conditioned stimuli in a conditioned suppression paradigm. Psychobiology, 18, 81-88.

Leung, H. T., \& Westbrook, R. F. (2010). Increased spontaneous recovery with increases in conditioned stimulus alone exposures. Journal of Experimental Psychology: Animal Behavior Processes, 36, 354367.

Lovibond, P. F., Preston, G. C., \& Mackintosh, N. J. (1984). Context specificity of conditioning, extinction, and latent inhibition. Journal of Experimental Psychology: Animal Behavior Processes, 10, 360-375. doi:10.1037/0097-7403.10.3.360

Lubow, R. E., Markman, R. E., \& Allen, J. (1968). Latent inhibition and classical conditioning of the rabbit pinna response. Journal of Comparative and Physiological Psychology, 66, 688-694.

Lubow, R. E., Schnur, P., \& Rifkin, B. (1976). Latent inhibition and conditioned attention theory. Journal of Experimental Psychology: Animal Behavior Processes, 2, 163-174. doi:10.1037/0097-7403.2. 2.163

Lubow, R. E., \& Siebert, L. (1969). Latent inhibition within the CER paradigm. Journal of Comparative and Physiological Psychology, $68,136-138$

Lubow, R. E., \& Weiner, I. (2010a). Issues in latent inhibition research and theory: An overview. In R. Lubow \& I. Weiner (Eds.), Latent inhibition: Cognition, neuroscience, and applications to schizophrenia (pp. 531-557). New York, NY: Cambridge University Press.

Lubow, R. E., \& Weiner, I. (2010b). Latent inhibition: Cognition, neuroscience, and applications to schizophrenia. Cambridge, UK: Cambridge University Press.

Nofrey, B. S., Ben-Shahar, O. M., \& Brake, W. G. (2008). Estrogen abolishes latent inhibition in ovariectomized female rats. Brain and Cognition, 66, 156-160.

Papini, M. R., Thomas, B. L., \& McVicar, D. G. (2002). Between-subject PREE and within-subject reversed PREE in spaced-trial extinction with pigeons. Learning and Motivation, 33, 485-509.

Peters, S. L., \& Joseph, M. H. (1993). Haloperidol potentiation of latent inhibition in rats: Evidence for a critical role at conditioning rather than pre-exposure. Behavioral Pharmacology, 4, 183-186.

Rankin, C. H. (2009). Introduction to special issue of neurobiology of learning and memory on habituation. Neurobiology of Learning and Memory, 92(2), 125-126.

Rosenberg, H., Holmes, N. M., Harris, J. A., \& Westbrook, R. F. (2011). Pre-exposure enhances recovery of conditioned responding after extinction. Learning \& Behavior, 39, 212-223.

Tai, C.-T., Cassaday, H. J., Feldon, J., \& Rawlins, J. N. P. (1995). Both electrolytic and excitotoxic lesions of nucleus accumbens disrupt latent inhibition of learning in rats. Neurobiology of Learning and Memory, 64, 36-48.

Tighe, T. J., \& Leaton, R. N. (1976). Habituation: Perspectives from child development, animal behavior, and neurophysiology. Hillsdale, $\mathrm{NJ}$ : Erlbaum.

Vervliet, B. (2013). Latent inhibition speeds up but weakens the extinction of conditioned fear in humans. Journal of Psychology and Psychotherapy, S7, 002. doi:10.4172/2161-0487.S7-002

Westbrook, R. F., \& Bouton, M. E. (2010). Latent inhibition and extinction: Their signature phenomena and the role of prediction error. In R. E. Lubow \& I. Weiner (Eds.), Latent inhibition: Cognition, neuroscience, and applications to schizophrenia (pp. 23-39). New York, NY: Cambridge University Press.

Westbrook, R. F., Jones, M. L., Bailey, G. K., \& Harris, J. A. (2000). Contextual control over conditioned responding in a latent inhibition paradigm. Journal of Experimental Psychology: Animal Behavior Processes, 26, 157-173. doi:10.1037/0097-7403.26.2.157 